\title{
A Comprehensive View on MRI Techniques for Imaging Blood-Brain Barrier Integrity
}

Citation for published version (APA):

Elschot, E. P., Backes, W. H., Postma, A. A., van Oostenbrugge, R. J., Staals, J., Rouhl, R. P. W., \& Jansen, J. F. A. (2021). A Comprehensive View on MRI Techniques for Imaging Blood-Brain Barrier Integrity. Investigative Radiology, 56(1), 10-19. https://doi.org/10.1097/RLI.0000000000000723

Document status and date:

Published: 01/01/2021

DOI:

10.1097/RLI.0000000000000723

Document Version:

Publisher's PDF, also known as Version of record

Document license:

Taverne

Please check the document version of this publication:

- A submitted manuscript is the version of the article upon submission and before peer-review. There can be important differences between the submitted version and the official published version of record.

People interested in the research are advised to contact the author for the final version of the publication, or visit the DOI to the publisher's website.

- The final author version and the galley proof are versions of the publication after peer review.

- The final published version features the final layout of the paper including the volume, issue and page numbers.

Link to publication

\footnotetext{
General rights rights.

- You may freely distribute the URL identifying the publication in the public portal. please follow below link for the End User Agreement:

www.umlib.nl/taverne-license

Take down policy

If you believe that this document breaches copyright please contact us at:

repository@maastrichtuniversity.nl

providing details and we will investigate your claim.
}

Copyright and moral rights for the publications made accessible in the public portal are retained by the authors and/or other copyright owners and it is a condition of accessing publications that users recognise and abide by the legal requirements associated with these

- Users may download and print one copy of any publication from the public portal for the purpose of private study or research.

- You may not further distribute the material or use it for any profit-making activity or commercial gain

If the publication is distributed under the terms of Article $25 \mathrm{fa}$ of the Dutch Copyright Act, indicated by the "Taverne" license above, 


\title{
A Comprehensive View on MRI Techniques for Imaging Blood-Brain Barrier Integrity
}

\author{
Elles P. Elschot, MSc, *广 Walter H. Backes, PhD, *†ई Alida A. Postma, MD, PhD, *† \\ Robert J. van Oostenbrugge, MD, PhD, †\$ Julie Staals, $M D, P h D, \neq \$$ \\ Rob P.W. Rouhl, MD, PhD, †+ and Jacobus F.A. Jansen, PhD*t/l
}

\begin{abstract}
The blood-brain barrier (BBB) is the interface between the blood and brain tissue, which regulates the maintenance of homeostasis within the brain. Impaired BBB integrity is increasingly associated with various neurological diseases. To gain a better understanding of the underlying processes involved in BBB breakdown, magnetic resonance imaging (MRI) techniques are highly suitable for noninvasive BBB assessment. Commonly used MRI techniques to assess BBB integrity are dynamic contrast-enhanced and dynamic susceptibility contrast MRI, both relying on leakage of gadolinium-based contrast agents. A number of conceptually different methods exist that target other aspects of the BBB. These alternative techniques make use of endogenous markers, such as water and glucose, as contrast media. A comprehensive overview of currently available MRI techniques to assess the BBB condition is provided from a scientific point of view, including potential applications in disease. Improvements that are required to make these techniques clinically more easily applicable will also be discussed.
\end{abstract}

Key Words: blood-brain barrier, magnetic resonance imaging, permeability, perfusion, neurodegenerative disorders, water exchange, glucoCEST

(Invest Radiol 2021;56: 10-19)

\section{Rationale}

In the last decade, a growing number of neurological conditions have been found to relate to an impaired integrity of the blood-brain barrier (BBB). ${ }^{1}$ To elucidate the role of the BBB in these diseases, robust, ideally noninvasive, in vivo methods are required that can measure even subtle changes and enable assessment of BBB disruption. Magnetic resonance imaging (MRI) provides a unique opportunity to noninvasively assess BBB impairment in various ways, as a number of conceptually different techniques have become available. ${ }^{2}$

The BBB is a physical barrier and functional interface between vasculature and parenchyma within the brain. By strictly regulating the transport of molecules from the capillaries into the neuronal tissue, and vice versa, the $\mathrm{BBB}$ is involved in the homeostasis maintenance of the central nervous system (CNS). Physically, the BBB mainly consists of endothelial cells that are connected via tight junctions, forming the walls of the capillaries in the brain. ${ }^{3}$ Transcellular transport over these

Received for publication June 29, 2020; and accepted for publication, after revision, August 1, 2020.

From the *Department of Radiology and Nuclear Medicine, Maastricht University Medical Center+; † School for Mental Health and Neuroscience, Maastricht University; \$Department of Neurology, Maastricht University Medical Center+; §CARIM School for Cardiovascular Diseases, Maastricht University, Maastricht; ||Department of Electrical Engineering, Eindhoven University of Technology, Eindhoven; and $\uparrow$ Academic Center for Epileptology Kempenhaeghe/Maastricht University Medical Center+, Maastricht, the Netherlands.

This work is part of the program Translational Research 2 with project number 446002509, funded by ZonMw/Epilepsiefonds.

Conflicts of interest and sources of funding: none declared.

This project has also received funding from the European Union's Horizon 2020 research and innovation program under grant agreement number 848109

Correspondence to: Jacobus F.A. Jansen, PhD, Department of Radiology and Nuclear Medicine, Maastricht University Medical Center, PO Box 5800, 6202 AZ Maastricht, the Netherlands. E-mail: jacobus.jansen@mumc.nl.

Copyright (C) 2020 Wolters Kluwer Health, Inc. All rights reserved.

ISSN: 0020-9996/21/5601-0010

DOI: $10.1097 /$ RLI.0000000000000723 capillary walls depends on the type of molecule and is controlled via passive diffusion, endothelial carrier proteins, and (receptor-mediated) transcytosis. $^{4}$

Impaired BBB integrity brings the CNS homeostasis out of balance, which may be because of or in response to pathological conditions in the brain. Alterations in transcellular transport, mainly caused by the disruption of tight junctions, lead to restricted removal of waste products. In addition, this allows possible extravasation of blood borne components leading to toxic levels in the brain tissue. The inflammatory response then eventually may lead to neuronal dysfunction. ${ }^{5}$ This ensures that alteration of the BBB permeability plays a crucial role in many diseases, including ischemic and hemorrhagic stroke, cerebral small vessel disease (cSVD), and brain cancer. Blood-brain barrier impairment is also considered as an early contributing factor to dysfunction of various processes involved in neurodegenerative disorders such as Alzheimer disease (AD) and multiple sclerosis (MS). In many cases, it is not clear whether BBB impairment is the cause or an effect of the disorder. Blood-brain barrier imaging has shown potential to better understand underlying processes in disorders such as stroke, cSVD, and $\mathrm{AD} .^{6-8}$ Therefore, the assessment of BBB impairment through imaging is of mechanistic and clinical importance.

The evaluation of BBB integrity for research purposes is commonly performed using MRI techniques that require administration of contrast agent (CA). ${ }^{9}$ It is the passive diffusion of a small molecular $\mathrm{CA}$ through the impaired BBB that is used as the detection mechanism. Two techniques that use a gadolinium-based CA are dynamic contrastenhanced (DCE) ${ }^{10}$ and dynamic susceptibility contrast (DSC) MRI. ${ }^{11,12}$ Dynamic contrast-enhanced MRI tracks the CA-induced signal changes on dynamic T1-weighted images, which makes it possible to obtain BBB permeability measures. Dynamic susceptibility contrast MRI measures (T2-weighted or) $\mathrm{T} 2 *$-weighted signal changes to evaluate brain perfusion properties. Other techniques that apply different contrast mechanisms, for example, using water exchange for assessment of BBB integrity ${ }^{13}$ or the use of D-glucose to quantify the role of BBB integrity in the cerebral energy metabolism, ${ }^{14}$ show possible value and target different features of BBB transport. Because these techniques do not make use of exogenous paramagnetic $\mathrm{CA}$, of which the safety has recently become of concern, imaging modalities without CA become more and more of interest. ${ }^{15,16}$ In addition to a variety of new contrast mechanisms, methods are continuously being improved to increase the sensitivity to subtle leakage and shorten scan times.

In this review, currently available MRI techniques and new developments for imaging of BBB integrity are explained from a scientific point of view. In addition, attention is being paid to current studies on potential applications in several diseases and required improvements to make the techniques clinically more feasible are discussed.

\section{Available MRI Methods}

In this section, we will discuss the available MRI methods and their applications in more details. For an overview, please refer to Table 1.

\section{Dynamic Contrast-Enhanced Imaging}

The most used technique to measure BBB impairment is DCEMRI. The technique makes use of a gadolinium-based contrast bolus 


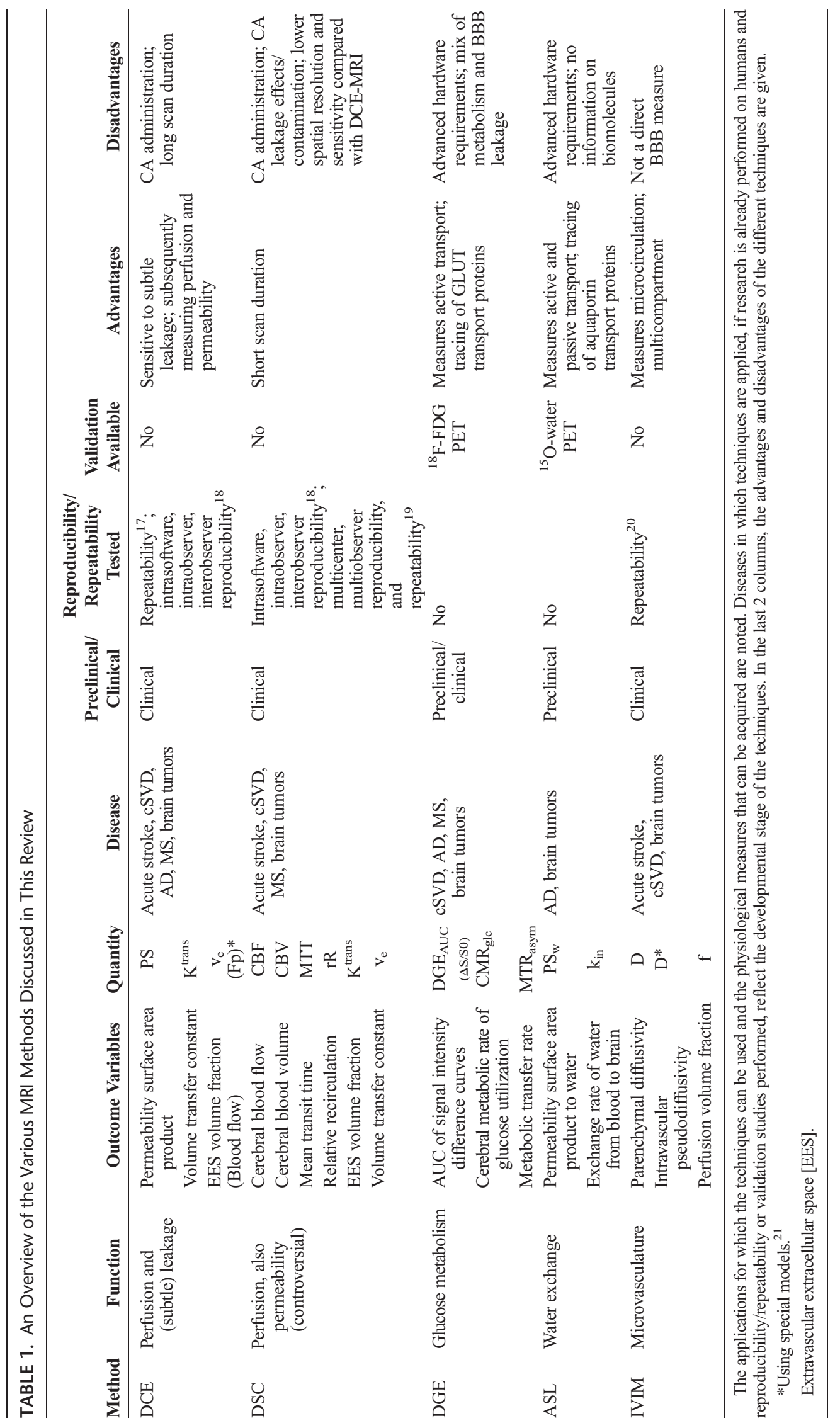




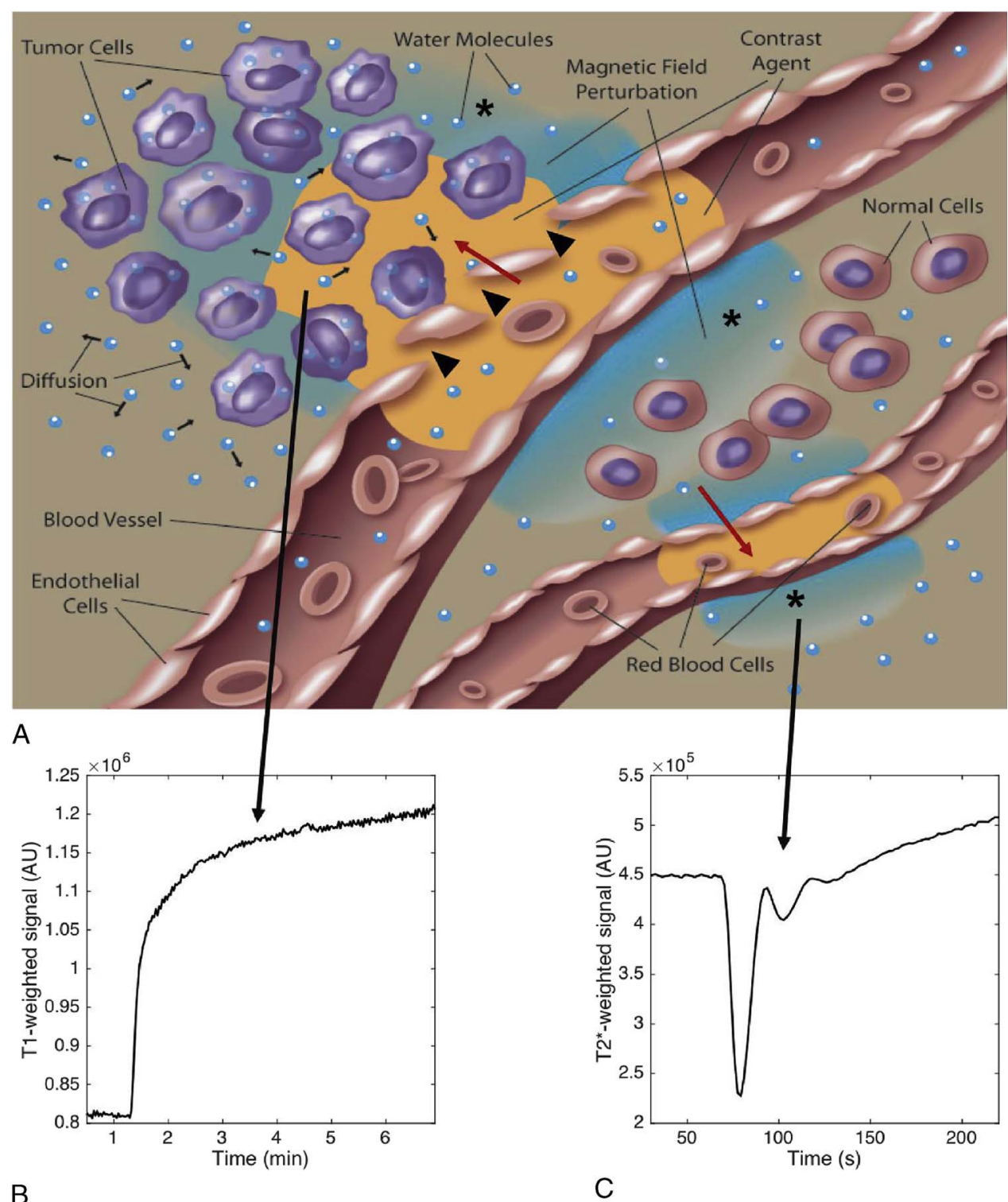

FIGURE 1. A, A representation of CA distribution when the BBB is intact versus impaired. When the BBB is intact (lower blood vessel), the CA can only interact with intravascular water (red arrow) resulting in minor signal changes. However, if the BBB is disrupted (top blood vessel, black triangles), the CA can interact with the water within the extravascular space (red arrow), substantially decreasing tissues T1 and increasing T1-weighted signal measured using DCE-MRI (B). The compartmentalization of CA in blood (lower blood vessel) or in the extravascular extracellular space (top blood vessel) gives rise to mesoscopic magnetic field gradients surrounding these compartments (as denoted by the asterisks). Diffusion of water through these fields (small black arrows) decreases (T2- weighted or) T2*-weighted signal, which can be measured using DSC-MRI (C). Obtained from Quarles et al. ${ }^{11}$

administration, after which a dynamic series of T1-weighted scans is acquired over time. Because of the T1 shortening effect of gadolinium, the concentration of CA can be obtained by assessing the signal intensity increases over time.

Considering the size and chemical structure, a gadolinium $\mathrm{CA}$ cannot pass the $\mathrm{BBB}$ in a healthy brain with intact BBB. However, when the integrity of the BBB is lost, degraded tight junctions make it possible for the CA to extravasate through the BBB (Fig. 1A). The accumulation of CA in the tissue's interstitial (ie, extravascular extracellular space [EES]) space will lead to increased T1-weighted signal intensity. Combining the tissue enhancement curves and blood supply of the CA by using pharmacokinetic model computations, the volume transfer constant $\mathrm{K}^{\text {trans }}$ as a surrogate measure of BBB permeability can be determined. ${ }^{21}$
Dynamic contrast-enhanced MRI is the only imaging technique currently available that allows for a direct assessment of BBB leakage and is sensitive for subtle leakage out of brain capillaries into the parenchyma. Nevertheless, to assess subtle and slow leakage, long measurements ( $>15$ minutes) are usually required. ${ }^{22}$ Reducing scan time to be suitable for a clinical setting while preserving sensitivity to subtle leakage is a point of attention for future research.

For the quantification of BBB leakage, pharmacokinetic modeling is generally used, in which the transfer rate is calculated by correcting for the supply of CA through the blood stream. For tumors, MS lesions, and infarctions, a 2-compartment model is commonly used, where the influx and backflux of the CA is considered to describe the exchange of the CA between blood and the interstitial space inside the tissue. ${ }^{21,23}$ However, for subtle leakage of CA into nonlesional 
tissue, the influx is very slow, the backflux can be ignored, and the graphical Patlak approach provides the most suitable leakage values. ${ }^{24}$

The sensitivity to detect subtle BBB leakage relies on the ability to detect low concentrations of gadolinium. Apart from the type of pulse sequence and signal-to-noise ratio, the change in relaxation rate determines the level of lowest detectable concentration. Because the $\mathrm{rl}$ and $\mathrm{r} 2$ relaxivity values of gadolinium-based CAs are approximately equal and the native $\mathrm{R} 1$ relaxation rate of brain tissue is much lower than R2, T1-weighted pulse sequences ought to be much more sensitive to measure subtle BBB leakage than T2-weighted pulse sequences.

\section{Dynamic Susceptibility Contrast Perfusion Imaging}

Also DSC-MRI relies on the administration of a paramagnetic CA. However, this technique uses T2-weighted or mostly T2*-weighted scans, which are sensitive to reductions in signal intensity caused by the magnetic susceptibility effects induced by relatively high concentrations of the CA in the blood stream (Fig. 1B). However, the T2* signal changes induced by the paramagnetic CA particles inside the blood vessel are not only confined in the blood space but extend somewhat to the region adjacent but outside the blood vessels (blooming effect). To obtain brain perfusion measures, the first-pass of the contrast bolus is measured using rapid imaging sequences. The short scan duration is one of the main advantages of DSC-MRI.

With hemodynamic modeling, the blood perfusion parameters cerebral blood volume (CBV), cerebral blood flow (CBF), and mean transit time (MTT) can be calculated from the DSC-MRI data by computational deconvolution methods applied to the tissue enhancement curve using the arterial blood concentration as input. Measurements of DSC perfusion have shown to be affected by extravasation of CA. Usually, the gadolinium concentration leaked into the parenchyma is very low and does not contribute to the $\mathrm{T} 2$ or $\mathrm{T} 2 *$ signal decreases, but in turn, it reverses the signal to an increase (T1 shine-through effect) and therefore contaminates the intended perfusion signal, especially at later time points after the first-pass peak. ${ }^{25-27}$ These effects influence the temporal dynamics of DSC-MRI signal, disturbs the linear behavior of $\mathrm{CA}$ relative to the relaxation rate, and results in an overestimation of $\mathrm{CBF}$ and $\mathrm{CBV}^{28,29}$ Image postprocessing methods have been proposed to compensate for the effects of CA leakage to the signal, of which the method by Weisskoff et $\mathrm{al}^{30}$ is most commonly used. This method subtracts the contribution of CA leakage from $\mathrm{T} 1$ enhancement by a linear fitting model. ${ }^{27}$

In some studies, additional DSC measures have been interpreted as a measure for BBB permeability. ${ }^{31-33}$ However, the reliability of this assumption has been questioned. Skinner et $\mathrm{al}^{34}$ demonstrated a lack of correlation between the permeability measure $\left(\mathrm{K}^{\text {trans }}\right)$ and perfusion measures $\left(\mathrm{K}_{\mathrm{a}}\right.$ and $\left.\mathrm{K}_{2}\right)$. Nevertheless, the volume transfer constant $\left(\mathrm{K}^{\text {trans }}\right)$ and EES volume fraction $\left(\mathrm{v}_{\mathrm{e}}\right)$ obtained with DSC-MRI were previously shown to be comparable with the same parameters obtained with DCE-MRI. ${ }^{35}$ It has to be considered though that these data were acquired in brain tumors where substantial leakage manifests. Whether DSC-MRI can also be applied to measure low concentration levels in pathologies where subtle leakage occurs is not yet known. To be able to correctly draw conclusions from BBB permeability parameters obtained from DSC-MRI, validation studies have to be performed. Nevertheless, detection of first-pass leakage to obtain perfusion parameters is of clinical value in therapy where strong leakage is expected, such as stroke and brain tumors.

\section{Glucose Chemical Exchange Saturation Transfer Imaging/Dynamic Glucose-Enhanced Imaging}

A recent innovation is the use of D-glucose as CA for MRI. D-Glucose is an endogenous metabolite and therefore biodegradable. Because glucose is the main energy source of the brain, examining glucose metabolism can give insight into the cerebral energy metabolism. ${ }^{36}$
After D-glucose bolus administration, the D-glucose signal can be measured over time using dynamic glucose-enhanced (DGE) MRI.

Detection of the signal is performed using chemical exchange saturation transfer (CEST) MRI, and therefore, the technique is also known as glucose chemical exchange saturation transfer (glucoCEST). The technique makes use of a frequency-selective radiofrequency pulse, saturating the protons from a molecule of interest. By looking at the exchange of saturated protons with protons from water molecules, the CEST signal can be obtained. Using this method, low concentrations of specific molecules can be measured, which cannot be detected by conventional MRI techniques. With 5 hydroxyl groups, the D-glucose molecule is well suited to be excited and measured with CEST-MRI (Fig. 2). By saturating the protons of the hydroxyl groups at a resonance frequency of $1.2 \mathrm{ppm}$, the exchange with bulk water protons can be determined, indirectly measuring the glucose metabolism.

Compared with gadolinium-based CAs, D-glucose not only enters the brain via diffusion through the impaired tight junctions but also passes the BBB actively via the GLUT1 glucose transporter protein. ${ }^{38}$ This entails a higher sensitivity of glucoCEST compared with gadolinium-based CA, and along with the smaller molecular weight of D-glucose compared with gadolinium compounds, this technique might be more sensitive to subtle leakage through an impaired BBB compared with DCE-MRI. Also, improved detection of BBB disruption might be feasible, although the influx by BBB transport needs to be balanced to the metabolic need of glucose. In addition, this technique carries lower safety risk and can be quantitatively validated by positron emission tomography (PET) using radioactively fluorine-labeled 2-deoxy-D-glucose $\left({ }^{18} \mathrm{~F}-\mathrm{FDG}\right) .{ }^{39}$

$\mathrm{Xu}$ et $\mathrm{al}^{40}$ was the first to translate glucoCEST MRI to humans at a field strength of $7 \mathrm{~T}$. The first results of recent studies at the clinical field strength of $3 \mathrm{~T}$ demonstrate the presence of glucoCEST enhancement in lesions of MS patients that were also found enhanced by DCE-MRI scans, indicating that glucose could possibly function as a CA for detection of BBB impairment in $\mathrm{MS} .{ }^{41}$ In some patients, where no gadolinium enhancement was observed, DGE enhancement was present, suggesting DGE-MRI to be more sensitive than DCE-MRI to BBB disruption, inflammation, or increased metabolic activity. However, the lower the field strength, the closer the saturation frequency of the hydroxyl protons comes to the resonance frequency of water (chemical shift reduces). It will therefore be more difficult to selectively saturate only the hydroxyl protons, which results in a background signal caused by direct water saturation and reduces the sensitivity of the technique. ${ }^{40}$ To reduce this sensitivity loss, B0-shimming is of high importance, and advanced MR hardware (with high power) is needed to apply a sufficiently selective radiofrequency pulse.

\section{Arterial Spin Labeling}

A large amount of transport pathways is involved in the exchange of water across the BBB (Fig. 3A). Measuring water exchange might lead to an interesting marker for detection of $\mathrm{BBB}$ impairment and can be performed by using arterial spin labeling (ASL). The technique might be sensitive to a wide range of $\mathrm{BBB}$ pathologies, because water is transported across the BBB by both passive and active diffusion mechanisms and is likely to be detectable at more subtle transfer rates because of the much smaller molecular weight and size of a water molecule compared with gadolinium CAs. ${ }^{13}$

Arterial spin labeling makes use of magnetic labeling (inverting the magnetization) of the incoming blood. ${ }^{13}$ After magnetic labeling, the magnetic spins flow through the brain into the imaging plane for a period defined by the postlabeling delay time. The signal difference between the 2 images obtained before and after the labeling of the inflowing blood represents the ASL signal. The location of the tagged magnetization can then be measured as a function of time, because of the differences in relaxation times and diffusivity between the 

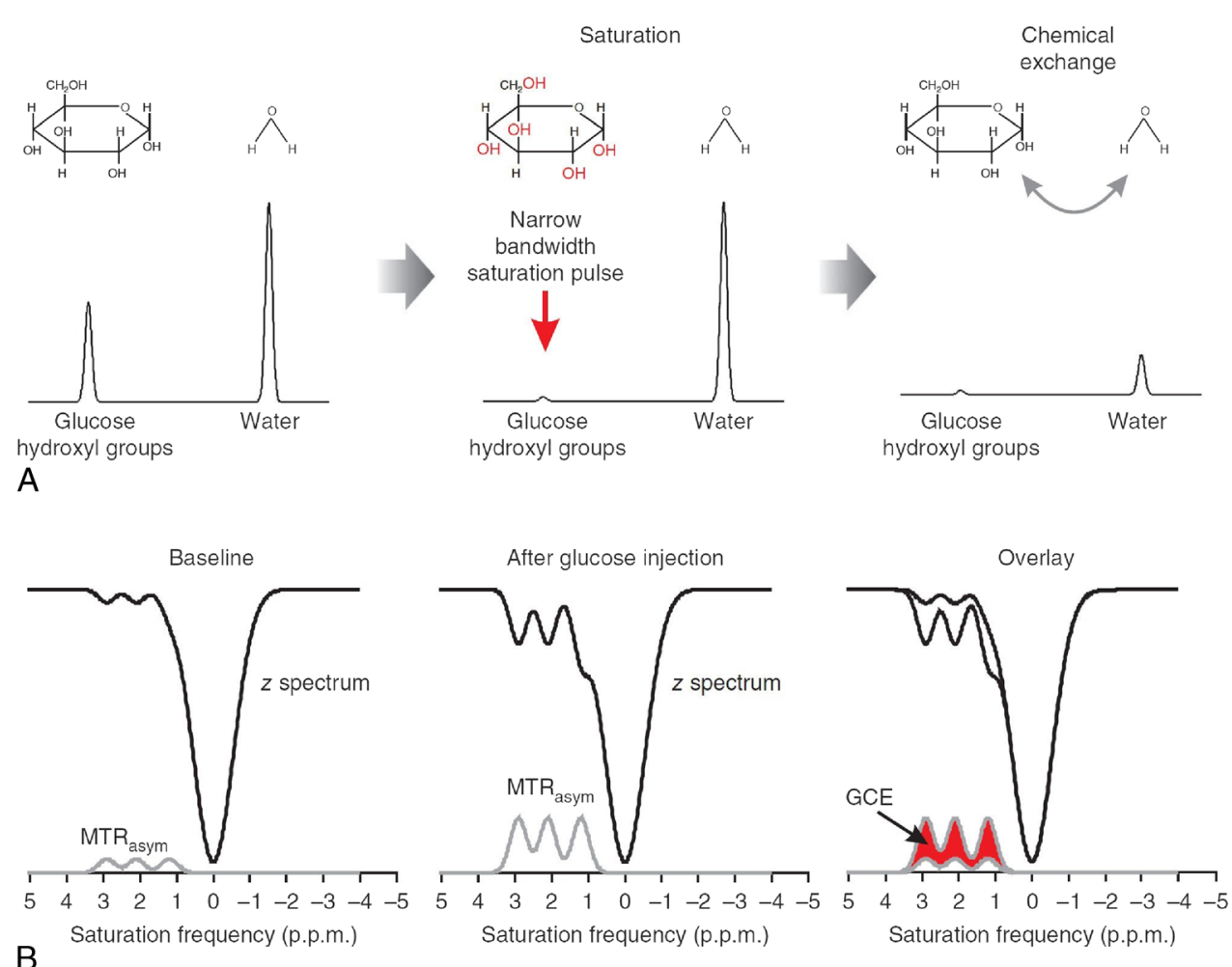

FIGURE 2. Schematic explanation of the principles underlying glucoCEST. A, Glucose and water pools are brought out of equilibrium by a saturation pulse at the resonance frequency of the molecule specific hydroxyl group (red). Proton exchange between the glucose hydroxyl groups and water reduces the measured signal. B, The CEST signal is usually expressed in a so-called Z-spectrum, where the water signal is measured as a function of saturation pulse frequency. The asymmetric magnetization transfer ratio $\left(\mathrm{MTR}_{\text {asym }}\right)$ is obtained by the difference signal of both sides of the water peak, centered at 0 ppm, representing the glucoCEST enhancement (GCE). Obtained from Walker-Samuel et al. ${ }^{37}$

intravascular and extravascular space compartments. Simultaneously, the labeled protons exchange the magnetization with the spins of bulk water, making it possible to measure the water exchange rates between the 2 compartments. With the knowledge of the fractional blood volume $\left(\mathrm{v}_{\mathrm{b}}\right)$ and the exchange rate of water from blood to brain $\left(\mathrm{k}_{\mathrm{in}}\right)$, the permeability surface area product of the BBB to water $\left(\mathrm{PS}_{\mathrm{w}}\right)$ can be calculated (Fig. 3B).
The relatively small differences in $\mathrm{T} 1$ relaxation time between blood and brain tissue and the involvement of location specific diffusion properties make the obtained ASL signal complicated. For all ASL-based measurements of water exchange, preknowledge of the arterial transit time (ATT) is needed, because the magnetic labeling is applied some time before the excitation of the imaging plane with the tissue of interest. In addition to the inflowing blood, the water signal

\section{Blood brain barrier transport pathways}

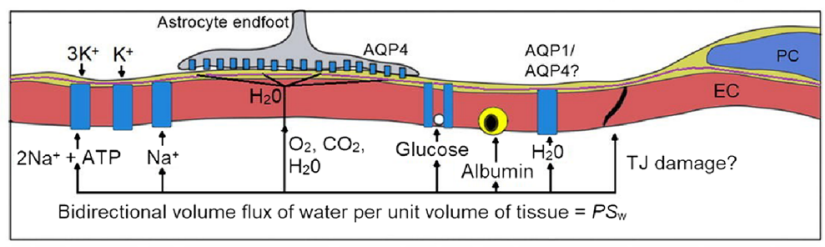

A

Water exchange rate, $k_{\text {in }}$

Permeability surface area product to water, $P S_{w}$

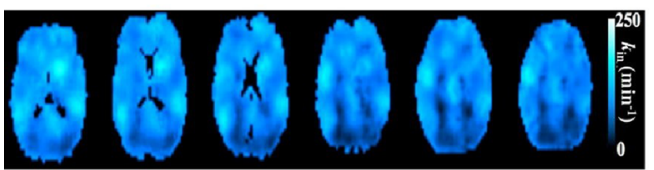

B

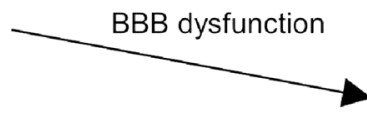

Magnetic resonance imaging (MRI)

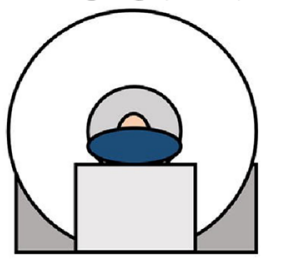

e.g. Contrast-enhanced MRI, diffusion weighted-ASL MRI, multi-echo time ASL MRI

FIGURE 3. A, Water movement over the BBB interface can occur through various transport pathways from blood to brain or vice-versa, and therefore has the potential to be altered in a range of BBB pathologies. B, Using multiple compartment modeling approaches measures as the water exchange rate $\left(\mathrm{k}_{\mathrm{in}}\right)$ and permeability surface area product to water $\left(\mathrm{PS}_{\mathrm{w}}\right)$ can be obtained. Obtained from Dickie et al. ${ }^{13}$ 
of the circulating cerebrospinal fluid (CSF) in the perivascular spaces will contribute to the ASL signal, making it difficult to trace whether the water signal measured is purely diffusion over the BBB.

Various methods have been developed to assess the water exchange across the BBB combining standard ASL with other magnetization preparation methods such as $\mathrm{T} 2$, diffusion, or magnetic transfer weighting. ${ }^{42-44}$ A combination of techniques might contribute to a better understanding of the influence of $\mathrm{T} 1, \mathrm{~T} 2$, and diffusion effects from the multiple compartments on the permeability quantities $\left(\mathrm{k}_{\mathrm{in}}\right.$ and $\left.\mathrm{PS}_{\mathrm{w}}\right)$. Improved multicompartment modeling approaches might be able to extract the water exchange signal because of the clearance system from the total signal to obtain only water exchange measures originating from the BBB. ${ }^{13}$

The potentially large sensitivity of this technique has already been proven in preclinical studies. Tiwari et $\mathrm{al}^{45}$ performed BBB permeability assessment with ASL and DCE simultaneously. This resulted in earlier detection of subtle BBB leakage and a higher level of measured BBB leakage compared with standard DCE-MRI. ${ }^{45}$ Also, the study of Dickie et $\mathrm{al}^{46}$ detected BBB breakdown in a rat model of AD using ASL, whereas no leakage could be detected with DCE-MRI. At this moment, the method developed by Wang et $\mathrm{al}^{43}$ provides the most robust assessment of cerebral perfusion in humans without the use of CA administration.

\section{Intravoxel Incoherent Motion Imaging}

Microcirculatory blood-flow can be studied using intravoxel incoherent motion (IVIM) imaging. For this technique, no contrast administration is needed as it is based on diffusion-weighted imaging methods. Diffusion-weighted imaging makes use of the free and restricted random motion of water molecules. ${ }^{47}$ The movement of free water in fluid is called thermal motion, caused by the influence of temperature, fluid viscosity, and molecular diffusivity. For water molecules inside capillaries, this motion is restricted by the vessel wall. These water molecules will flow along with the bloodstream in the network of capillaries, which is termed pseudodiffusion. In IVIM imaging, these 2 different types moving water molecules are separated by describing the signal in a biexponential model. This way, parameters for parenchymal diffusivity (D), intravascular pseudodiffusivity (D*), and blood volume perfusion fraction (f) can be obtained. ${ }^{48}$ Although these parameters are not direct measures of BBB permeability, the microvascular metrics $f$ and $D^{*}$ are perfusion-related and therefore thought to provide information about BBB integrity. For example, a preclinical comparison study with DCE-MRI in an oncology setting demonstrated moderate agreement between $\mathrm{f}$ and $\mathrm{K}^{\text {trans }} \cdot{ }^{49}$ Whether this relationship also holds in subtle BBB leakage remains to be investigated.

\section{MRI Studies to Detect BBB Impairment in Disease}

\section{Acute Stroke}

Hemorrhagic stroke is caused by vessel rupture, which goes together with a disrupted BBB. An altered BBB integrity after stroke can express itself in several ways and is related to the severity of the condition. ${ }^{50}$ In ischemic stroke, the BBB gets disrupted because of the oxidative stress shortly after onset of ischemia. Disruption of tight junctions is the major cause of increased BBB permeability after stroke, allowing water to flow into the extravascular space to form edema. Edema can also accumulate in the presence of an intact BBB because of imbalance of active transport mechanisms. ${ }^{51,52}$

To measure increases in BBB permeability because of stroke, DCE-MRI is the most sensitive clinically applied technique. ${ }^{53-55} \mathrm{Be}-$ cause DCE-MRI is able to detect subtle leakage, it has potential in early determination of appropriate treatments and monitoring of therapeutic effects. Prediction of pathological consequences after stroke, such as hemorrhagic transformation and poststroke epilepsy, might become possible using DCE-MRI. ${ }^{56,57}$ Dynamic glucose-enhanced and water exchange imaging techniques are potential applications for the detection of early alterations in active transport over the $\mathrm{BBB}$, because these methods are able to specifically measure active transport mechanisms and might be more sensitive than DCE-MRI early in the pathologic cascade. Water exchange imaging using a diffusion-weighted ASL method was shown to be sensitive to BBB permeability increases in an ischemic stroke rat model, which was confirmed by simultaneous DCE imaging. ${ }^{45}$

An alternative method to obtain microvascular perfusion and diffusion information in ischemic stroke patients is IVIM ${ }^{58}$ Edema leads to a decrease of Brownian motion and diffusion space in the extracellular space, which may result in a reduction of $\mathrm{D}^{*}, \mathrm{f}$, and also the product $\mathrm{fD}^{*} .^{59-61}$ The effect of diffusion restriction of water molecules also enables to determine the core and penumbra region. ${ }^{62}$ Although IVIM does not directly refer to BBB permeability, detection of impaired microcirculation inside the ischemic tissue and the penumbra might be helpful in explaining pathological conditions during stroke and might function as a marker of stroke severity at baseline. ${ }^{63,64}$

Caution is needed when interpreting IVIM parameters in stroke, because assumptions made in modeling approaches might no longer be valid in pathologic tissue, where pathophysiological processes vary over time. ${ }^{60}$ The microvascular compartment might be impaired in stroke, and acute tissue swelling in early stages of stroke might affect the obtained measures.

\section{Cerebral Small Vessel Disease}

Cerebral small vessel disease is a term covering multiple pathologies of the small vessels in the brain, and BBB impairment is a feature in all variants of this disease. ${ }^{8}$ Dynamic contrast-enhanced MRI has been identified as the most sensitive technique for cSVD to determine the subtle BBB leakage. ${ }^{17,65}$ Because of the small vessel size and subtle leakage rate, quantitative measurements for this purpose using DSC-MRI are challenging. ${ }^{8}$

Water exchange imaging is a promising venue in cSVD research. The fact that no CA is needed makes it sensitive to low BBB permeability, because the size and molecular weight of water is smaller compared with gadolinium. Shao et al ${ }^{66}$ performed diffusion-weighted ASL imaging to measure water exchange in patients with high risk for cSVD and showed that BBB water permeability could function as an early biomarker for cSVD.

Cerebral small vessel disease patients are known to have alterations in the brain microvasculature and also the parenchyma. Therefore, IVIM might be a useful imaging technique in cSVD. A first study in cSVD patients using IVIM was performed by Wong et al, ${ }^{67}$ where interestingly an increase in perfusion fraction was observed. The same effect was found in a study where IVIM was used to assess the hippocampal microvasculature in patients with type 2 diabetes, a known risk factor for cSVD. ${ }^{68}$ Possible explanations could be vasodilation of the microvessels, enlarged perivascular spaces, increased vessel tortuosity, or reduced $\mathrm{BBB}$ integrity. More research is needed to gain a better understanding of the complex microenvironment and the contribution of blood and other brain fluids to these results.

\section{Alzheimer Disease}

Cerebrovascular impairment seems to play a major role in the development of $\mathrm{AD}{ }^{69}$ Subtle BBB leakage already occurs at early-onset $\mathrm{AD}$, so sensitive imaging techniques are needed to be able to measure $\mathrm{BBB}$ permeability changes early in $\mathrm{AD} .^{7,70}$ Dual-time resolution DCE imaging has been shown to measure subtle leakage in the cortical gray matter of patients with early AD. ${ }^{71}$

Impairment of the BBB initiates multiple proinflammatory and cytotoxic events involved in $\mathrm{AD}$, such as alterations in the clearing mechanisms of the brain. This might contribute to the accumulation 
of amyloid beta peptides, which is one of the hallmarks of AD. ${ }^{72}$ Amyloid beta also reduces the expression of glucose transporters at the $\mathrm{BBB}$, even before the onset of $\mathrm{AD}$ symptoms. ${ }^{38}$ In most studies, glucose metabolism in the brain of $\mathrm{AD}$ patients is measured using ${ }^{18}$ F-FDG PET. ${ }^{73-75}$ Dynamic glucose-enhanced might be an alternative method to study altered glucose transport over the BBB in $\mathrm{AD}$ pathology. ${ }^{76}$

The multiple pathological processes involved in $\mathrm{AD}$ might also disturb the role of the $\mathrm{BBB}$ in water exchange. ${ }^{77}$ Water exchange MRI is therefore another interesting technique for measuring BBB impairment in AD. Multicompartment modeling approaches are needed as alterations in the cleaning (ie, glymphatic) system might also contribute to the water exchange signal.

\section{Multiple Sclerosis}

Impairment of the BBB is increasingly being seen as a valuable biomarker in MS, because it occurs at the earliest stages of disease. ${ }^{78,79}$ The inflammatory feature of MS increases the expression of cytokines on the endothelial cells, which leads to the upregulation of cell adhesion molecules that facilitate transcellular transport of leukocytes over the $\mathrm{BBB} .{ }^{80}$ In addition, alterations in the expression of tight junctions increase BBB dysfunction. ${ }^{81}$

Pathological changes of BBB integrity in MS can be detected early in disease using DCE-MRI, especially in the normal-appearing white matter, which is the most prone region to develop MS lesions. ${ }^{24,82,83}$ Furthermore, imaging of glucose transport may function as a sensitive technique for detection of active MS lesions, because contrast-enhanced lesions also showed positive DGE signal. ${ }^{41}$ However, it should be noted that glucose consumption by leukocytes and macrophages is increased in MS lesions, possibly resulting in a combined signal of metabolic needs and BBB disruption. Impaired BBB function also seems to result in reduced water exchange in relapsing remitting MS patients, which was suggested to be caused by inactive ion pumps because of alterations in metabolic activity of neurons. ${ }^{84}$

\section{Primary Brain Tumors and Cerebral Metastasis}

The BBB in tumor tissue is also called the blood-tumor barrier because it has specific characteristics leading to alterations in permeability not only in the brain but also the spinal cord (ie, CNS barrier), including impaired tight junction complexes and altered expression of transporter proteins. ${ }^{85}$ Traditionally, enhancement of brain tumors is considered to be cause by increased vascular permeability as a result of $\mathrm{BBB}$ breakdown in high-grade tumors in contrast to nonenhancing low-grade tumors, although more specific molecular markers of tumor aggressiveness are nowadays used to predict tumor progression and medical outcome. For low-grade tumors, the BBB also plays an important role for the transportation and accumulation of specific proteins.

Impairment of the blood-tumor barrier and the BBB of the surrounding tissue is tumor type specific, and therefore, imaging parameters differ per tumor type. ${ }^{85}$ This makes DCE and DSC imaging techniques important in the diagnosis, monitoring of progression, and prediction of medical outcome in cancer. ${ }^{11}$

Particular tumor types, for example, breast cancer tumors that form metastases in the brain, express more GLUT1 transport proteins. ${ }^{86}$ Alterations in transcellular transport proteins result in vasogenic edema. This increases the interest in application of advanced imaging techniques such as glucose and water exchange imaging in brain cancer. Xu et $\mathrm{al}^{87}$ already measured enhanced glucose levels in the tumor region using DGE-MRI. Previously, Wang et al ${ }^{88}$ detected increased water exchange rates compared with healthy tissue in a single patient with grade II oligodendroglioma. These first results from human studies are promising, but much more research is needed to confirm the conclusions drawn.

\section{Required Future Developments}

Improvements of existing MRI techniques and the advent of new contrast mechanisms to measure BBB integrity (see Table 1) increase the knowledge about the pathophysiological processes underlying BBB impairment in various neurological conditions and diseases. The various techniques are measuring different aspects of BBB impairment. However, what techniques serve best or add valuable information for various pathological conditions remains to be shown. Terms to express BBB integrity are used interchangeably, making interpretation of results somewhat confusing. To correctly interpret measures obtained with different techniques, a more complete understanding of ongoing physiological processes is needed. So far, the only method that can be used to measure pure BBB permeability is DCE-MRI, because CA-enhanced T1-weighted signal in brain tissue is directly related to BBB leakage (after correction of the blood content). By increasing the scan durations, it should be possible to detect even more subtle levels of leakage. ${ }^{22}$ Contrarily, DSC-MRI primarily assesses blood perfusion rather than permeability. Modeling approaches have been published to obtain first-pass permeability measures from DSC data. Because many biological processes are affected in various ways during the time frame of disease, validation is needed for the use of these models in specific pathologic conditions. Because of the short scan time of DSC-MRI and the external influences on the T2/T2*-weighted signal, this technique is less sensitive compared with DCE-MRI. Alterations in active transport over the BBB can noninvasively be assessed using DGE-MRI, because glucose energy metabolism is regulated by GLUT1 transporters. Arterial spin labeling-based techniques are suitable for a combination of active and passive transport mechanisms over the $\mathrm{BBB}$, because both water exchange facilitated by aquaporins and transcellular water diffusion are contributing to the ASL signal. Analyzing signal contributions from the vasculature and parenchyma using IVIM imaging provides microvascular diffusivity measures, possibly linked to BBB integrity. Combining ASL and IVIM might also be promising to assess BBB integrity, because intravascular and extravascular water signal could be differentiated, which might lead to a gain in understanding of diffusion properties. ${ }^{88,89}$

Assessment of BBB integrity is, beyond stroke and brain tumors, mostly applied in clinical research settings these days. Techniques that are ready for implementation in the clinic are DCE-MRI and DSCMRI. These techniques might be a valuable tool for assessment of pathology and prediction of treatment response. However, to make these techniques applicable for clinical use, some improvements have to be made. First of all, standardized MRI protocols need to become available for image acquisition and data analysis to be able to compare studies with large study populations over various sites. ${ }^{8}$ One of the points of attention in techniques using CAs is the determination of the optimal dose, timing, portioning, and rate for contrast administration. ${ }^{90}$ Development of commercial software for image analysis would improve the ease of use for clinicians. Also, reproducibility studies are needed for longitudinal research to assess the dynamic nature of the BBB.

Attention should be paid to recent findings in gadolinium-based CA usage disclosing the entry of CA into the CSF via the glymphatic pathways through the CNS. ${ }^{91}$ When infiltration of gadolinium-based $\mathrm{CA}$ into the CSF in any other way than via an impaired $\mathrm{BBB}$ can be validated, research on CA-based methods assessing BBB integrity should also consider these alternative glymphatic pathways.

The techniques described in this review that do not require $\mathrm{CA}$ administration are still in a preliminary phase of development. Further advances of imaging hardware, such as higher power and improved B0-shimming methods, are required to provide sufficient sensitivity and temporal resolution for the use of these techniques in the clinic. 
More studies on human subjects are needed to establish test-retest repeatability, and validation of sensitivity to subtle BBB breakdown in pathology has to be proven before standardization.

Validation experiments are hard to perform as no golden "in vivo" standard is currently available for BBB integrity. An available application to obtain a validation measure for $\mathrm{BBB}$ integrity is lumbar puncture for determination of CSF/serum albumin ratio $\left(\mathrm{Q}_{\mathrm{alb}}\right) .{ }^{92} \mathrm{Be}-$ cause albumin is not synthesized in the brain and cannot pass an intact $\mathrm{BBB}$, the CSF albumin index is directly related to albumin diffusion across an impaired BBB. Because of the invasive procedure, this method is not preferable. A promising application for validation of active transport measures over the BBB could be the combination of PETMRI, ${ }^{93}$ as a direct comparison between water exchange or glucose metabolism and activity of corresponding transporters can be made using PET. Particularly, PET tracers can be detected at extremely low concentrations (picomolar ranges), far below the detection limit of gadolinium with MRI (micromolar ranges). A recent study in AD patients found a significant correlation between reduced $\mathrm{Q}_{\mathrm{alb}}$ and decreased levels of ${ }^{18}$ F-FDG uptake, which might be related to BBB dysfunction, showing the possible application of PET as a validation technique for BBB integrity. ${ }^{94}$

The extended knowledge of BBB status could be used in future applications, for example, to predict the risk of metastasis or advanced stages of cerebrovascular disease. Also, the interest in the role of the BBB in neurological drug uptake is currently increasing. ${ }^{95}$ The use of MR-guided focused ultrasound techniques yielding targeted BBB disruption might improve drug delivery to the brain. ${ }^{96}$ Other topics of interest for future research could be the effect of healthy aging on the $\mathrm{BBB},{ }^{97}$ linking cognition to $\mathrm{BBB}$ integrity, and assessment of $\mathrm{BBB}$ characteristics of the normal-appearing tissue surrounding the tumor.

This review has demonstrated the value of advanced MRI techniques for the assessment of BBB integrity in pathology. A number of emerging MRI techniques that address properties of the BBB impairment in different ways become available. Although processes involved in impaired BBB integrity are becoming clearer, there are still many knowledge gaps to be filled in. By expanding the possibilities for imaging techniques to assess the BBB, additional insights might be obtained, contributing to a better understanding of $\mathrm{BBB}$ impairment in brain disease.

\section{REFERENCES}

1. Sweeney MD, Zhao Z, Montagne A, et al. Blood-brain barrier: from physiology to disease and Back. Physiol Rev. 2019;99:21-78.

2. Veksler R, Shelef I, Friedman A. Blood-brain barrier imaging in human neuropathologies. Arch Med Res. 2014;45:646-652.

3. Abbott NJ, Patabendige AAK, Dolman DEM, et al. Structure and function of the blood-brain barrier. Neurobiol Dis. 2010;37:13-25.

4. Abbott NJ, Rönnbäck L, Hansson E. Astrocyte-endothelial interactions at the blood-brain barrier. Nat Rev Neurosci. 2006;7:41-53.

5. Varatharaj A, Galea I. The blood-brain barrier in systemic inflammation. Brain Behav Immun. 2017;60:1-12.

6. Jiang X, Andjelkovic AV, Zhu L, et al. Blood-brain barrier dysfunction and recovery after ischemic stroke. Prog Neurobiol. 2018;163-164:144-171.

7. Raja R, Rosenberg GA, Caprihan A. MRI measurements of blood-brain barrier function in dementia: a review of recent studies. Neuropharmacology. 2018;134: 259-271.

8. Thrippleton MJ, Backes WH, Sourbron S, et al. Quantifying blood-brain barrier leakage in small vessel disease: review and consensus recommendations. Alzheimers Dement. 2019;15:840-858.

9. Montagne A, Toga AW, Zlokovic BV. Blood-brain barrier permeability and gadolinium: benefits and potential pitfalls in research. JAMA Neurol. 2016;73:13.

10. Heye AK, Culling RD, Valdés Hernández MDC, et al. Assessment of blood-brain barrier disruption using dynamic contrast-enhanced MRI. A systematic review. Neuroimage Clin. 2014;6:262-274.

11. Quarles CC, Bell LC, Stokes AM. Imaging vascular and hemodynamic features of the brain using dynamic susceptibility contrast and dynamic contrast enhanced MRI. Neuroimage. 2019;187:32-55.
12. Jahng GH, Li KL, Ostergaard L, et al. Perfusion magnetic resonance imaging: a comprehensive update on principles and techniques. Korean J Radiol. 2014; 15:554.

13. Dickie BR, Parker GJM, Parkes LM. Measuring water exchange across the blood-brain barrier using MRI. Prog Nucl Magn Reson Spectrosc. 2020;116: 19-39.

14. Xu X, Chan KWY, Knutsson L, et al. Dynamic glucose enhanced (DGE) MRI for combined imaging of blood-brain barrier break down and increased blood volume in brain cancer. Magn Reson Med. 2015;74:1556-1563.

15. Harvey HB, Gowda V, Cheng G. Gadolinium deposition disease: a new risk management threat. J Am Coll Radiol. 2020;17:546-550.

16. Runge VM. Critical questions regarding gadolinium deposition in the brain and body after injections of the gadolinium-based contrast agents, safety, and clinical recommendations in consideration of the EMA's Pharmacovigilance and risk assessment committee recommendation for suspension of the marketing authorizations for 4 linear agents. Invest Radiol. 2017;52:317-323.

17. Wong SM, Jansen JFA, Zhang CE, et al. Measuring subtle leakage of the blood-brain barrier in cerebrovascular disease with DCE-MRI: test-retest reproducibility and its influencing factors. J Magn Reson Imaging. 2017;46:159-166.

18. Conte GM, Castellano A, Altabella L, et al. Reproducibility of dynamic contrast-enhanced MRI and dynamic susceptibility contrast MRI in the study of brain gliomas: a comparison of data obtained using different commercial software. Radiol Med. 2017;122:294-302.

19. Smits M, Bendszus M, Collette S, et al. Repeatability and reproducibility of relative cerebral blood volume measurement of recurrent glioma in a multicentre trial setting. Eur J Cancer. 2019;114:89-96.

20. Wong SM, Backes WH, Zhang CE, et al. On the reproducibility of inversion recovery intravoxel incoherent motion imaging in cerebrovascular disease. $\mathrm{Am} \mathrm{J}$ Neuroradiol. 2018;39:226-231.

21. Sourbron SP, Buckley DL. Classic models for dynamic contrast-enhanced MRI NMR Biomed. 2013;26:1004-1027.

22. Van De Haar HJ, Jansen JFA, Jeukens CRLPN, et al. Subtle blood-brain barrier leakage rate and spatial extent: considerations for dynamic contrast-enhanced MRI: considerations. Med Phys. 2017;44:4112-4125.

23. Tofts PS, Brix G, Buckley DL, et al. Estimating kinetic parameters from dynamic contrast-enhanced T1-weighted MRI of a diffusable tracer: standardized quantities and symbols. J Magn Reson Imaging. 1999;10:223-232.

24. Cramer SP, Larsson HBW. Accurate determination of blood-brain barrier permeability using dynamic contrast-enhanced T1-weighted MRI: a simulation and in vivo study on healthy subjects and multiple sclerosis patients. J Cereb Blood Flow Metab. 2014;34:1655-1665.

25. Paulson ES, Schmainda KM. Comparison of dynamic susceptibility-weighted contrast-enhanced MR methods: recommendations for measuring relative cerebral blood volume in brain tumors. Radiology. 2008;249:601-613.

26. Quarles CC, Gochberg DF, Gore JC, et al. A theoretical framework to mode DSC-MRI data acquired in the presence of contrast agent extravasation. Phys Med Biol. 2009;54:5749-5766.

27. Boxerman JL, Schmainda KM, Weisskoff RM. Relative cerebral blood volume maps corrected for contrast agent extravasation significantly correlate with glioma tumor grade, whereas uncorrected maps do not. AJNR Am J Neuroradiol. 2006; 27:859-867.

28. Blockley NP, Jiang L, Gardener AG, et al. Field strength dependence of R and R2* relaxivities of human whole blood to ProHance, Vasovist, and deoxyhemoglobin. Magn Reson Med. 2008;60:1313-1320.

29. Marstrand JR, Rostrup E, Rosenbaum S, et al. Cerebral hemodynamic changes measured by gradient-echo or spin-echo bolus tracking and its correlation to changes in ICA blood flow measured by phase-mapping MRI. J Magn Reson Imaging. 2001;14:391-400.

30. Weisskoff RM, Boxerman JL, Sorensen AG, et al. Simultaneous blood volume and permeability mapping using a single Gd-based contrast injection. In: Proceedings of the 2nd Annual Meeting of ISMRM, San Francisco vol. 279. Concord, CA International Society for Magnetic Resonance in Medicine; 1994.

31. Provenzale JM, Wang GR, Brenner T, et al. Comparison of permeability in high-grade and low-grade brain tumors using dynamic susceptibility contrast MR imaging. Am J Roentgenol. 2002;178:711-716.

32. Thornhill RE, Chen S, Rammo W, et al. Contrast-enhanced MR imaging in acute ischemic stroke: $\mathrm{T} 2 *$ measures of blood-brain barrier permeability and their relationship to T1 estimates and hemorrhagic transformation. Am J Neuroradiol. 2010;31:1015-1022.

33. Leigh R, Jen SS, Varma DD, et al. Arrival time correction for dynamic susceptibility contrast MR permeability imaging in stroke patients. PLoS One. 2012 7:e52656

34. Skinner JT, Moots PL, Ayers GD, et al. On the use of DSC-MRI for measuring vascular permeability. Am J Neuroradiol. 2016;37:80-87. 
35. Quarles CC, Gore JC, Xu L, et al. Comparison of dual-echo DSC-MRI- and DCEMRI-derived contrast agent kinetic parameters. Magn Reson Imaging. 2012;30: 944-953.

36. Kuzawa CW, Chugani HT, Grossman LI, et al. Metabolic costs and evolutionary implications of human brain development. Proc Natl Acad Sci U S A. 2014;111: 13010-13015.

37. Walker-Samuel S, Ramasawmy R, Torrealdea $\mathrm{F}$, et al. In vivo imaging of glucose uptake and metabolism in tumors. Nat Med. 2013;19:1067-1072.

38. Patching SG. Glucose transporters at the blood-brain barrier: function, regulation and gateways for drug delivery. Mol Neurobiol. 2017;54:1046-1077.

39. Yang FY, Chang WY, Chen JC, et al. Quantitative assessment of cerebral glucose metabolic rates after blood-brain barrier disruption induced by focused ultrasound using FDG-MicroPET. Neuroimage. 2014;90:93-98.

40. Xu X, Yadav NN, Knutsson L, et al. Dynamic glucose-enhanced (DGE) MRI: translation to human scanning and first results in glioma patients. Tomography. 2015;1:105-114.

41. Xu X, Bhargava P, Sehgal A, et al. GlucoCEST MRI of blood brain barrier disruption in multiple sclerosis-first human experiments and initial results. ECTRIMS Online Library.2019; P1788.

42. Gregori J, Schuff N, Kern R, et al. T2-based arterial spin labeling measurements of blood to tissue water transfer in human brain. J Magn Reson Imaging. 2013;37: 332-342.

43. Wang DJJ, Alger JR, Qiao JX, et al. The value of arterial spin-labeled perfusion imaging in acute ischemic stroke: comparison with dynamic susceptibility contrast-enhanced MRI. Stroke. 2012;43:1018-1024.

44. Silva AC, Zhang W, Williams DS, et al. Estimation of water extraction fractions in rat brain using magnetic resonance measurement of perfusion with arterial spin labeling. Magn Reson Med. 1997;37:58-68.

45. Tiwari YV, Lu J, Shen Q, et al. Magnetic resonance imaging of blood-brain barrier permeability in ischemic stroke using diffusion-weighted arterial spin labeling in rats. J Cereb Blood Flow Metab. 2017;37:2706-2715.

46. Dickie BR, Vandesquille M, Ulloa J, et al. Water-exchange MRI detects subtle blood-brain barrier breakdown in Alzheimer's disease rats. Neuroimage. 2019; 184:349-358.

47. Federau C. Intravoxel incoherent motion MRI as a means to measure in vivo perfusion: a review of the evidence. NMR Biomed. 2017;30:1-15.

48. Paschoal AM, Leoni RF, dos Santos AC, et al. Intravoxel incoherent motion MRI in neurological and cerebrovascular diseases. NeuroImage Clin. 2018;20: 705-714.

49. Yang X, Xiao X, Lu B, et al. Perfusion-sensitive parameters of intravoxel incoherent motion MRI in rectal cancer: evaluation of reproducibility and correlation with dynamic contrast-enhanced MRI. Acta Radiol. 2019;60:569-577.

50. Prakash R, Carmichael ST. Blood-brain barrier breakdown and neovascularization processes after stroke and traumatic brain injury. Curr Opin Neurol. 2015;28: $556-564$.

51. Stokum JA, Gerzanich V, Simard JM. Molecular pathophysiology of cerebral edema. J Cereb Blood Flow Metab. 2016;36:513-538.

52. Chen YJ, Wallace BK, Yuen N, et al. Blood-brain barrier KCa3.1 channels. Stroke. 2015;46:237-244.

53. Merali Z, Huang K, Mikulis D, et al. Evolution of blood-brain-barrier permeability after acute ischemic stroke. PLoS One. 2017;12:e0171558.

54. Villringer K, Sanz Cuesta BE, Ostwaldt AC, et al. DCE-MRI blood-brain barrier assessment in acute ischemic stroke. Neurology. 2017;88:433-440.

55. Kassner A, Mandell DM, Mikulis DJ. Measuring permeability in acute ischemic stroke. Neuroimaging Clin N Am. 2011;21:315-325.

56. Huang WYY, Wu G, Li JJJ, et al. Prediction of reperfusion-associated hemorrhagic transformation using dynamic contrast-enhanced imaging in a rat stroke model. J Comput Assist Tomogr. 2015;39:787-793.

57. van Vliet EA, Otte WM, Gorter JA, et al. Longitudinal assessment of blood-brain barrier leakage during epileptogenesis in rats. A quantitative MRI study. Neurobiol Dis. 2014;63:74-84.

58. Jansen JFA, Wong SM, Backes WH. IVIM MRI: a window to the pathophysiology underlying cerebral small vessel disease. In: Intravoxel Incoherent Motion (IVIM) MRI. Singapore: Jenny Stanford Publishing; 2018:85-98.

59. Yao Y, Zhang S, Tang X, et al. Intravoxel incoherent motion diffusion-weighted imaging in stroke patients: initial clinical experience. Clin Radiol. 2016;71:938. e11-938.e16.

60. Federau $\mathrm{C}$, Sumer $\mathrm{S}$, Becce $\mathrm{F}$, et al. Intravoxel incoherent motion perfusion imaging in acute stroke: initial clinical experience. Neuroradiology. 2014;56:629-635.

61. Hu LBB, Hong N, Zhu WZZ. Quantitative measurement of cerebral perfusion with intravoxel incoherent motion in acute ischemia stroke: initial clinical experience. Chin Med J (Engl). 2015;128:2565-2569.

62. Suo S, Cao M, Zhu W, et al. Stroke assessment with intravoxel incoherent motion diffusion-weighted MRI. NMR Biomed. 2016;29:320-328
63. Liu Z, Xiao X. The use of multi b values diffusion-weighted imaging in patients with acute stroke. Neuroradiology. 2013;55:371-376.

64. Zhu G, Federau C, Wintermark M, et al. Comparison of MRI IVIM and MR perfusion imaging in acute ischemic stroke due to large vessel occlusion. Int J Stroke. 2020;15:332-342.

65. Zhang CE, Wong SM, van de Haar HJ, et al. Blood-brain barrier leakage is more widespread in patients with cerebral small vessel disease. Neurology. 2017;88: $426-432$

66. Shao X, Ma SJ, Casey M, et al. Mapping water exchange across the blood-brain barrier using 3D diffusion-prepared arterial spin labeled perfusion MRI. Magn Reson Med. 2019;81:3065-3079.

67. Wong SM, Zhang CE, van Bussel FCG, et al. Simultaneous investigation of microvasculature and parenchyma in cerebral small vessel disease using intravoxel incoherent motion imaging. NeuroImage Clin. 2017;14:216-221.

68. Van Bussel FC, Backes WH, Hofman PA, et al. On the interplay of microvasculature, parenchyma, and memory in type 2 diabetes. Diabetes Care. 2015;38: 876-882.

69. Sweeney MD, Montagne A, Sagare AP, et al. Vascular dysfunction-the disregarded partner of Alzheimer's disease. Alzheimers Dement. 2019;15: $158-167$.

70. van de Haar HJ, Burgmans S, Jansen JFA, et al. Blood-brain barrier leakage in patients with early Alzheimer disease. Radiology. 2016;281:527-535.

71. Haar HJ, Jansen JFA, Jeukens CRLPN, et al. Subtle blood-brain barrier leakage rate and spatial extent: considerations for dynamic contrast-enhanced MRI Med Phys. 2017;44:4112-4125.

72. Zenaro E, Piacentino G, Constantin G. The blood-brain barrier in Alzheimer's disease. Neurobiol Dis. 2017;107:41-56.

73. Fan Z, Aman Y, Ahmed I, et al. Influence of microglial activation on neurona function in Alzheimer's and Parkinson's disease dementia. Alzheimers Dement. 2015;11:608.e7-621.e7.

74. Winkler EA, Nishida Y, Sagare AP, et al. GLUT1 reductions exacerbate Alzheimer's disease vasculo-neuronal dysfunction and degeneration Nat Neurosci. 2015;18:521-530.

75. Hunt A, Schönknecht P, Henze M, et al. Reduced cerebral glucose metabolism in patients at risk for Alzheimer's disease. Psychiatry Res. 2007;155:147-154.

76. Huang J, van Zijl PCM, Han X, et al. Altered D-glucose in brain parenchyma and cerebrospinal fluid of early Alzheimer's disease detected by dynamic glucose-enhanced MRI. Sci Adv. 2020;6:eaba3884.

77. Anderson VC, Lenar DP, Quinn JF, et al. The blood-brain barrier and microvascular water exchange in Alzheimer's disease. Cardiovasc Psychiatry Neurol. 2011; 2011:1-9.

78. Cramer SP, Modvig S, Simonsen HJ, et al. Permeability of the blood-brain barrier predicts conversion from optic neuritis to multiple sclerosis. Brain. 2015;138: 2571-2583.

79. Vos CMP, Geurts JJG, Montagne L, et al. Blood-brain barrier alterations in both focal and diffuse abnormalities on postmortem MRI in multiple sclerosis. Neurobiol Dis. 2005;20:953-960.

80. Ortiz GG, Pacheco-Moisés FP, Macías-Islas MÁ, et al. Role of the blood-brain barrier in multiple sclerosis. Arch Med Res. 2014;45:687-697.

81. Spencer JI, Bell JS, DeLuca GC. Vascular pathology in multiple sclerosis: reframing pathogenesis around the blood-brain barrier. J Neurol Neurosurg Psychiatry. 2018;89:42-52.

82. Xiong H, Yin P, Li X, et al. The features of cerebral permeability and perfusion detected by dynamic contrast-enhanced magnetic resonance imaging with Patlak model in relapsing-remitting multiple sclerosis. Ther Clin Risk Manag. 2019;15: 233-240.

83. Ingrisch $\mathrm{M}$, Sourbron $\mathrm{S}$, Morhard $\mathrm{D}$, et al. Quantification of perfusion and permeability in multiple sclerosis: dynamic contrast-enhanced MRI in 3D at 3T. Invest Radiol. 2012;47:252-258.

84. Rooney WD, Li X, Sammi MK, et al. Mapping human brain capillary water lifetime: high-resolution metabolic neuroimaging. NMR Biomed. 2015;28:607-623.

85. Arvanitis CD, Ferraro GB, Jain RK. The blood-brain barrier and blood-tumour barrier in brain tumours and metastases. Nat Rev Cancer. 2020;20:26-41.

86. Yonemori K, Tsuta K, Ono M, et al. Disruption of the blood brain barrier by brain metastases of triple-negative and basal-type breast cancer but not HER2/neu-positive breast cancer. Cancer. 2010;116:302-308.

87. Xu X, Sehgal AA, Yadav NN, et al. D-glucose weighted chemical exchange saturation transfer (glucoCEST)-based dynamic glucose enhanced (DGE) MRI at 3T: early experience in healthy volunteers and brain tumor patients. Magn Reson Med. 2020;84:247-262.

88. Wang J, Fernández-Seara MA, Wang S, et al. When perfusion meets diffusion: in vivo measurement of water permeability in human brain. $J$ Cereb Blood Flow Metab. 2007;27:839-849. 
89. Zhang X, Ingo C, Teeuwisse WM, et al. Comparison of perfusion signal acquired by arterial spin labeling-prepared intravoxel incoherent motion (IVIM) MRI and conventional IVIM MRI to unravel the origin of the IVIM signal. Magn Reson Med. 2018;79:723-729.

90. Boxerman JL, Quarles CC, Hu LS, et al. Consensus recommendations for a dynamic susceptibility contrast MRI protocol for use in high-grade Gliomas. Neuro Oncol. 2020;1-10.

91. Deike-Hofmann K, Reuter J, Haase R, et al. Glymphatic pathway of gadolinium-based contrast agents through the brain: overlooked and misinterpreted. Invest Radiol. 2019;54:229-237.

92. Reiber H, Peter JB. Cerebrospinal fluid analysis: disease-related data patterns and evaluation programs. J Neurol Sci. 2001;184:101-122.
93. Kilbourn MR. Small molecule PET tracers for transporter imaging. Semin Nucl Med. 2017;47:536-552.

94. Chiaravalloti A, Fiorentini A, Ursini F, et al. Is cerebral glucose metabolism related to blood-brain barrier dysfunction and intrathecal IgG synthesis in Alzheimer disease? A ${ }^{18}$ F-FDG PET/CT study. Medicine (Baltimore). 2016;95:e4206.

95. Pandit R, Chen L, Götz J. The blood-brain barrier: physiology and strategies for drug delivery. Adv Drug Deliv Rev. 2019;S0169-409X:30238-30238.

96. Fisher DG, Price RJ. Recent advances in the use of focused ultrasound for magnetic resonance image-guided therapeutic nanoparticle delivery to the central nervous system. Front Pharmacol. 2019;10:1348.

97. Verheggen ICM, de Jong JJA, van Boxtel MPJ, et al. Increase in blood-brain barrier leakage in healthy, older adults. GeroScience. 2020;42:1183-1193. 